\title{
Slow quasigeostrophic unstable modes of a lens vortex in a continuously stratified flow
}

\author{
Hai Yen Nguyen, Bach Lien Hua*, Richard Schopp and Xavier Carton
}

IFREMER, Laboratoire de Physique des Oceans, BP 70, 29280 Plouzané , France

*: Corresponding author : Bach Lien Hua, email address : Bach.Lien.Hua@ifremer.fr

\begin{abstract}
:
This work addresses the linear dynamics underlying the formation of density interfaces at the periphery of energetic vortices, well outside the vortex core, both in the radial and axial directions. We compute numerically the unstable modes of an anticyclonic Gaussian vortex lens in a continuously stratified rotating fluid. The most unstable mode is a slow mode, associated with a critical layer instability located at the vortex periphery. Although the most unstable disturbance has a characteristic vertical scale which is comparable to the vortex height, interestingly, the critical levels of the successively fastest growing modes are closely spaced at intervals along the axial direction that are much smaller than the vortex height.
\end{abstract}

Keywords: Quasigeostrophic model ; Vortices ; Stratified flow ; Critical level ; Flow instability

\section{Introduction}

Persistent layering, defined here as the vertical stacking of sharp interfaces in temperature and density, has been recently evidenced at the periphery of energetic oceanic vortices using seismic imaging of the water column (Biescas et al. 2008, Menesguen et al. 2009a, Papenberg et al. 2010, Quentel et al. 2010). Typical values of the observed vertical scales involved in the layering is in the range of a few meters up to about 100 meters. What is novel is the documentation that such long-lived layering is abundant not only at the lateral periphery of vortices, as emphasized in early observational work (Armi et al. 1989), but is even stronger both aloft and below the eddy core, at the vertical periphery of the vortex. Another striking observation is the lateral spatial coherence of the layering which can extend over distances comparable to the vortex horizontal size for several tens of kilometers. These two characteristic properties of the layering, its localization at the periphery of oceanic intra-thermocline vortices and its large lateral coherence, are the focus of the present study. Here, we present adiabatic dynamical arguments for sharp interfaces formation that depart from previously invoked rationales for layering that invoke, either different diffusive properties of temperature and salt in the ocean(e.g. Ruddick and Richards 2003), or which require an inertial instability of the flow (e.g. Menesguen et al. 2009b).

We build on the study by Benilov (2003) which dealt with the possibility of a slow destabilization of oceanic vortices through critical level instabilities at the lateral periphery of vortices based on an asymptotic analysis of a thin vortex in a two-layer configuration. In the present paper we explore numerically how his results on critical level instabilities can generalize to the case of an intrathermocline vortex lens in a continuously stratified fluid.

The above cited work is part of an abundant litterature on oceanic vortices stability which started with Gent and McWilliams (1986), Flierl (1988), and Helfrich and Send (1988), and were motivated 
by observational data such as the one of Armi et al. (1989). Other observations concerned the surfaceintensified Gulf Stream Ring vortices Olson (1991), whose longevity, and thus robustness to splitting mechanisms by baroclinic or barotropic instability, have been studied by Dewar and Killworth (1995), Dewar et al. (1999), Dritschel and de la Torre Juarez (1996), Drijfhout (2003), Katsman et al. (2003), Reinaud and Carton (2009).

We shall restrict ourselves here to the case of quasigeostrophic dynamics (Pedlosky 1996), the roles of ageostrophic and divergent waves dynamics on vortex stability have been discussed in the two-layer shallow water studies of Baey and Carton (2002) and Benilov and Flanagan (2008) and will also be addressed in forthcoming numerical studies using continuous primitive and also Boussinesq equations.

\section{The governing equations}

The conservation of quasigeostrophic potential vorticity (Pedlosky 1996) reads

$$
\frac{\partial Q}{\partial t}+J(\Psi, Q)=0
$$

where $\Psi$ is the streamfunction, $J$ the horizontal Jacobian operator and $Q$ the potential vorticity defined as

$$
Q \equiv \nabla^{2} \Psi+f^{2}\left(\frac{\Psi_{z}}{N^{2}}\right)_{z}+f
$$

where $z$ is the vertical coordinate, $f$ is the constant Coriolis parameter and $N$ is the Brunt-Väisälä profile.

Denoting by $\left(\psi^{\prime}, Q^{\prime}\right)$ perturbations of the streamfunction and potential vorticity around the base state $\bar{\psi}, \bar{Q}$, the linearization of equation (1) yields

$$
\frac{\partial Q^{\prime}}{\partial t}+J\left(\bar{\psi}, Q^{\prime}\right)+J\left(\psi^{\prime}, \bar{Q}\right)=0
$$

Henceforth, we restrict the work to axisymmetric mean flows, with $\bar{\psi}$ a function of the radial and vertical coordinates $r$ and $z$ only. $\bar{\psi}$ is assumed to be three-dimensionally compact and $\rightarrow 0$ as $r \rightarrow \infty$ or $|z| \rightarrow \infty$. The perturbations are assumed to be normal modes of the form

$$
\psi^{\prime}=\psi(r, z) \mathrm{e}^{\mathrm{i} m(\theta-c t)},
$$

where $m$ and $c$ are the azimuthal wavenumber and angular phase speed. Equation (3) leads to

$$
\left(c-\frac{\bar{\psi}_{r}}{r}\right)\left[\frac{1}{r}\left(r \psi_{r}\right)_{r}-\frac{m^{2}}{r^{2}} \psi+\frac{f^{2}}{N^{2}} \psi_{z z}\right]+\frac{1}{r} \psi \bar{Q}_{r}=0,
$$

as here $N$ is assumed to be constant.

The base state is chosen to represent a lens-like vortex, whose lateral and vertical scales are respectively denoted as $L$ and $H$ and are used as length scales for the nondimensionalization :

$$
r \longrightarrow r / L, \quad z \longrightarrow z / H, \quad \psi \longrightarrow \psi / f L^{2} .
$$

Specifically, the generic shape which is used corresponds to an anticyclonic vortex lens

$$
\bar{\psi}(r, z)=\frac{R o}{4}\left(1-b z^{2}\right) \mathrm{e}^{-\left(r^{2}+z^{2}\right)} .
$$

In (7), when parameter $b=0$ the lens has a simple Gaussian shape, while $b \neq 0$ corresponds to a case with counterrotating flows above and below the vortex core. The flow amplitude is set by the Rossby number 
$R o\left(\equiv\left|\zeta_{0}\right| / f\right.$, where $\zeta_{0}$ is the relative vorticity of the vortex at its center). Introducing the Burger number of the base state

$$
B u \equiv \frac{N^{2} H^{2}}{f^{2} L^{2}}
$$

the ambiant potential vorticity $\bar{Q}$ is

$$
\bar{Q}(r, z)=(r \bar{\psi})_{r} / r+B u^{-1} \bar{\psi}_{z z}+1 .
$$

The boundary conditions (see Appendix A) are

$$
\psi \propto\left\{\begin{array} { l } 
{ r ^ { | m | } \quad \text { as } \quad r \longrightarrow 0 , } \\
{ r ^ { - | m | } \quad \text { as } \quad r \longrightarrow \infty , }
\end{array} \quad \psi _ { z } = 0 \quad \text { at } \quad \left\{\begin{array}{l}
z=z_{\text {top }}, \\
z=z_{\text {bottom }} .
\end{array}\right.\right.
$$

We thus need to solve

$$
\left[-\frac{1}{r} \bar{Q}_{r}+\frac{1}{r} \bar{\psi}_{r}\left(\frac{1}{r} \partial_{r}+\partial_{r r}-m^{2} / r^{2}+B u^{-1} \partial_{z z}\right)\right] \psi=c\left(\frac{1}{r} \partial_{r}+\partial_{r r}-m^{2} / r^{2}+B u^{-1} \partial_{z z}\right) \psi
$$

with the boundary conditions (10), which correspond to a generalized eigenvalue problem of the form $\boldsymbol{A} \boldsymbol{\Psi}=c \boldsymbol{B} \boldsymbol{\Psi}$, where $\boldsymbol{A}$ and $\boldsymbol{B}$ are differential operators.

\section{$3 \quad$ Stability results}

Equation (11) and the associated boundary conditions (10) have been discretized horizontally and vertically using a resolution of $100 \times 100$ grid points for $r=0, . ., r_{\text {max }}=6$ and $z=0, . ., z_{\text {top }}$, using centered finite differences and symmetric (resp. antisymmetic) conditions at $z=0$. The system has been solved by a direct generalized eigenvalue so lver QZ method.

\subsection{Counter-and co-rotating lens}

Previous studies, e.g. Baey and Carton (2002), Benilov (2003), have demonstrated the dominance of low azimuthal modes in the destabilization of baroclinic vortices. The results of the stability analysis as a function of the Burger number of the lens are displayed for azimuthal modes $m=1$ and $m=2$ in figure 1, for lenses with only co-rotating flow (a) for $b=0$, and with counter-rotating flows along the vertical direction for (b) and (c), corresponding respectively to $b=0.7$, and $b=1.4$. Since equation (5) is invariant for $z \longrightarrow-z$, solutions can be either symmetric or antisymmetric with respect to $z=0$ and both cases have been considered for $m=1$ (see also the discussion in Gent and McWilliams 1986). Figure 1(a) for a Gaussian lens with only co-rotating flow confirms published results for the lens stability, namely that wider lenses (small $B u$ ) are more unstable and that, in general, the most unstable mode for baroclinic instability corresponds to an azimuthal mode $m=2$. For tall lenses $(B u>1)$, i.e. lenses whose height exceeds the rescaled length scale $(f / N) L$, the most unstable mode corresponds to an antisymmetric $m=1$ mode, thus recovering the results that barotropic instability prevails for tall columnar vortices embedded in a rotating stratified flow, established by Gent and McWilliams (1986) (see also Dritschel and de la Torre Juarez 1996). Growth rates differ by an order of magnitude between co- and counterrotating flows (compare figures 1(a) and 1(b)), as initially recognized by Dewar and Killworth (1995) and the destabilizing influence of counter-rotating flows has been checked to vary uniformly with parameter $b$ in equation (7). The increased instability of such counter-rotating vortices fully agrees with their lack of oceanic observations.

The lowest azimuthal modes $m=1,2$ have also been consistently found to be the fastest growing modes in the stability results reported in Tychensky (1999) for both a 3-layer and a 5-layer system. A general 


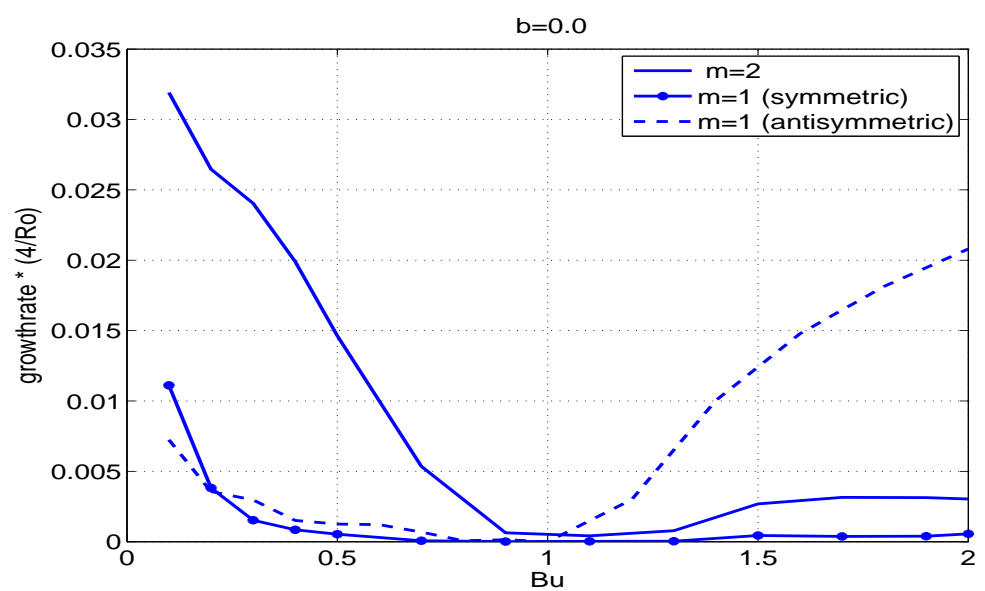

(a)

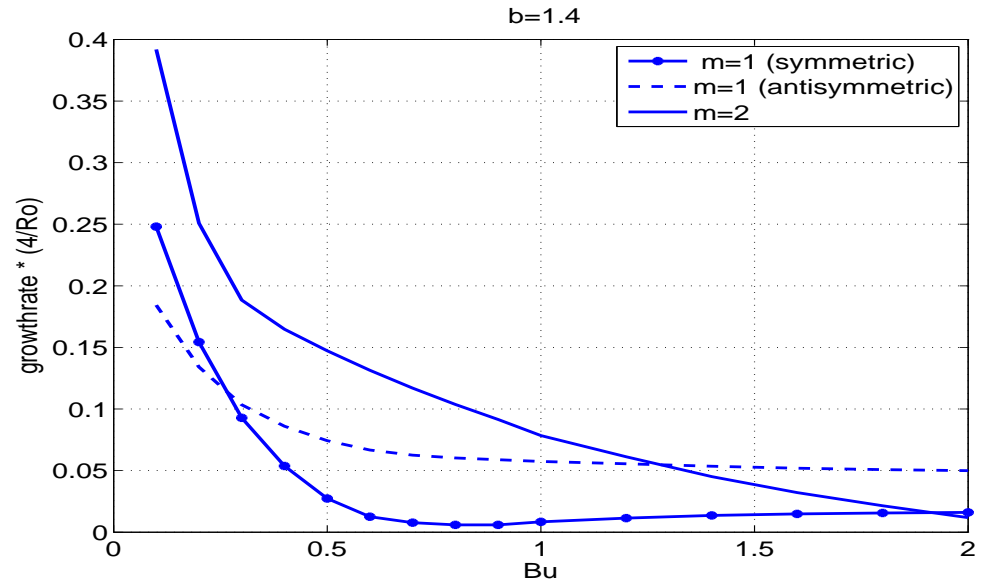

(b)

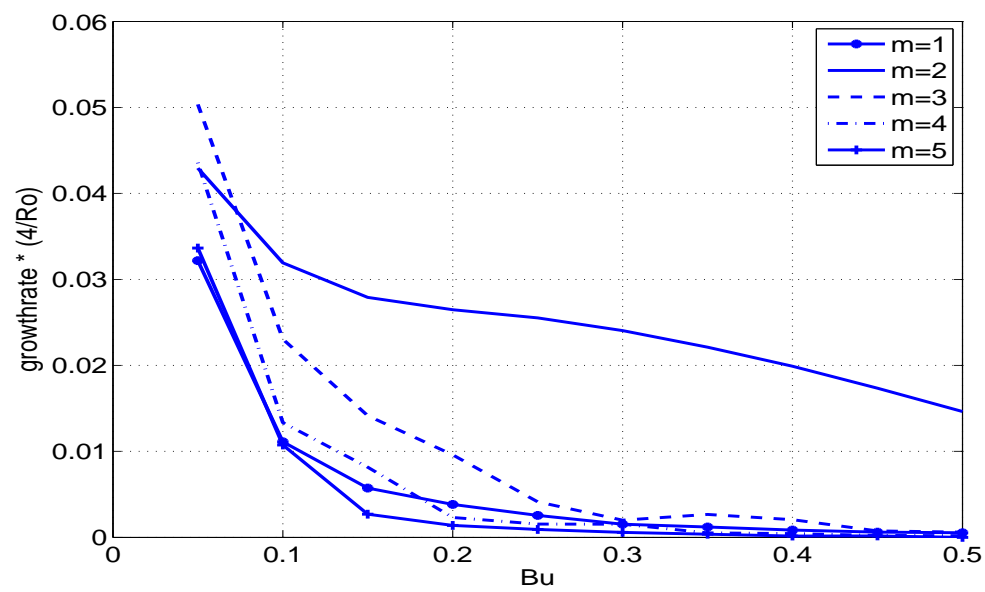

(c)

Figure 1. Growth rate of the fastest growing eigenmode for (a) $\mathrm{b}=0.0$, (b) $\mathrm{b}=1.4$ in a domain such that $z_{\text {top }}=-z_{\text {bottom }}=6$. [Note the factor of 10 in ordinate scale between (a) and (b)]. (c) Growth rate of azimuthal modes up to $m=5$ for wide Gaussian vortices with $b=0$.

discussion of the influence of the radial profile of the vortex core on the azimutal modes selection for the barotropic case is given in figure 2 of Balmforth et al. (2001). For the baroclinic instability of a wide gaussian vortex $(B u<1$ and $b=0$ in equation (7)), the dominance of azimuthal mode $m=2$ holds for $0.1<B u<1$. However in the limit of vanishing $B u$ higher azimuthal modes become dominant, as shown in figure 1(c), e.g. the cross-over of the growth rate curves of azimuthal modes $m=2$ and $m=3$ occurs 


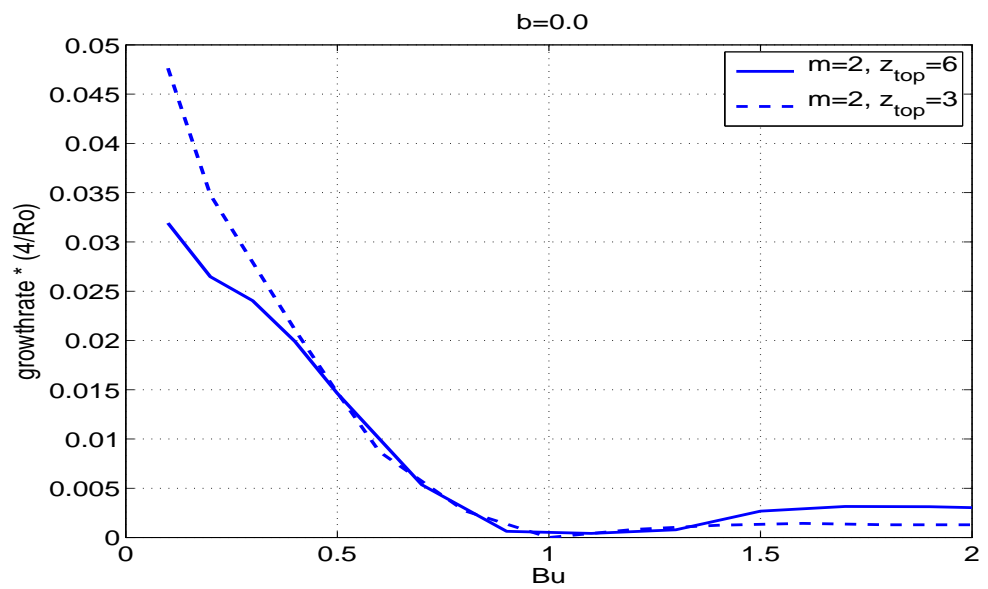

Figure 2. Growth rate variation with thinness of lens for a domain such that $z_{t o p}=-z_{\text {bottom }}=6$ (continuous line) and $z_{\text {top }}=-z_{\text {bottom }}=3$ (dash line).

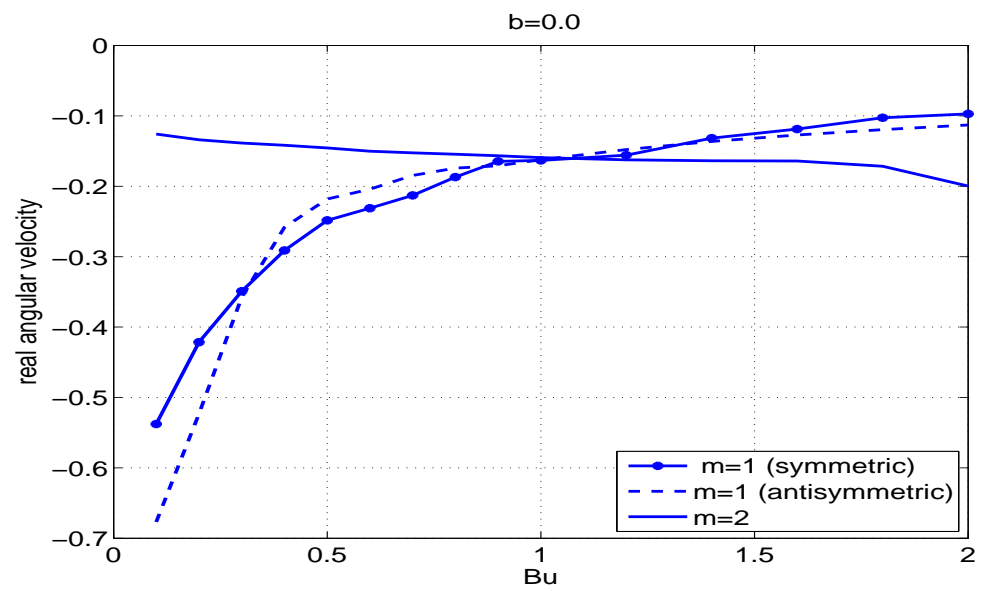

Figure 3. Real part of the angular velocity $\operatorname{Re}\{c\}$ of the leading unstable mode for $\mathrm{b}=0.0$ for a domain such that $z_{\text {top }}=-z_{\text {bottom }}=6$.

for $B u=0.075$, while $m=4$ will dominate over $m=2$ for $B u<0.05$.

The two-layer study of Benilov (2003) showed that stability results strongly depend on the relative thinness of the eddy with respect to the lower layer thickness. This sensitivity has been examined in figure 2 for the case of the azimuthal mode $m=2$ by examining the influence of varying the total depth of the domain and contrasting the two cases with $z_{\text {top }}=-z_{\text {bottom }}=6$ (continuous line) and $z_{\text {top }}=-z_{\text {bottom }}=3$ (dash line). Figure 2 confirms the result that thin lenses are more stable than thicker ones, but the sensitivity is found to remain relatively small in the case of the base state of equation (7).

\subsection{Critical level localization}

As elaborated by the asymptotic analysis of Benilov (2003), the preferred destabilization of wide baroclinic vortices (small $B u$ ) occurs preferentially through "slow" unstable modes, whose real part of the complex angular frequency is small when compared to the maximal swirl angular velocity of the vortex core. This explains why unstable modes are associated to critical levels that are located at the vortex periphery since the swirl velocity of the vortex decreases with radial distance from the vortex center and thus highlights the crucial importance of the vortex periphery for its stability. Figure 3 gives the distribution as a function of $B u$ of the real angular phase speed of the leading unstable mode for a co-rotating vortex 


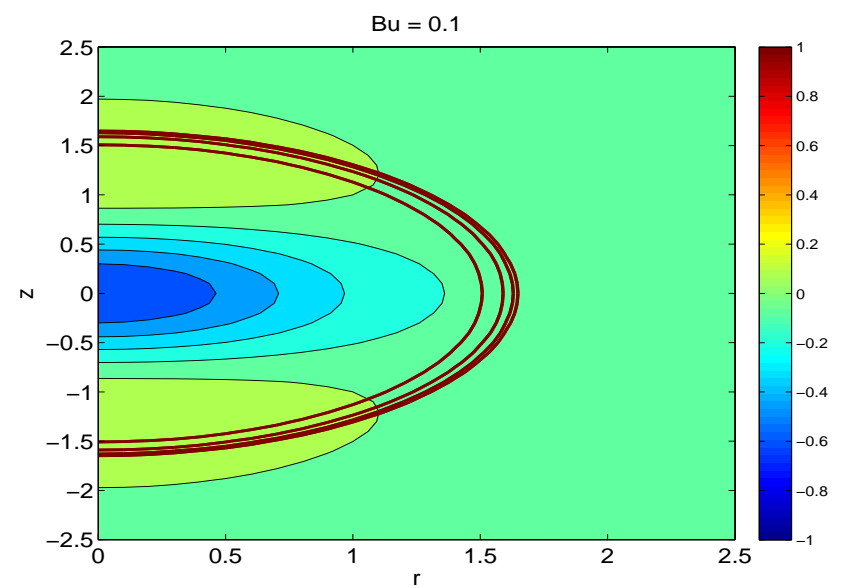

(a)

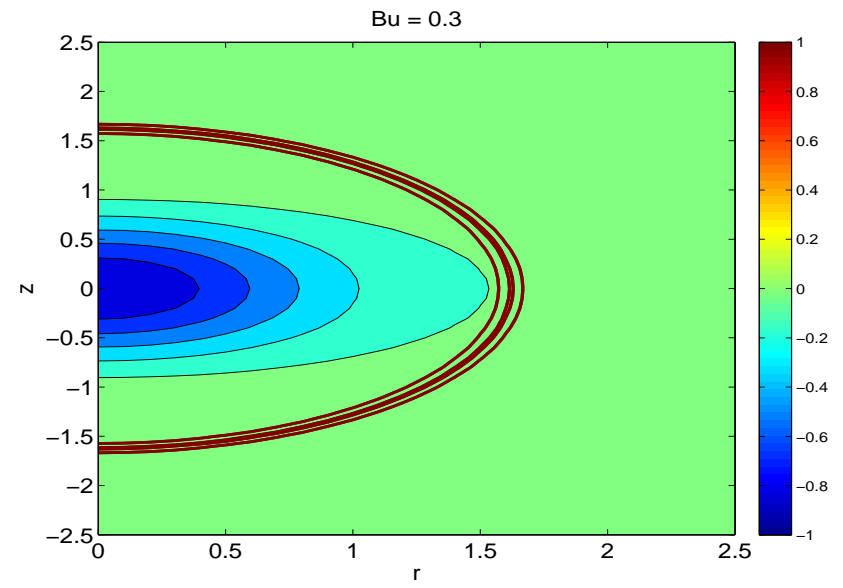

(b)

Figure 4. Critical levels of the four most unstable eigenmodes on top of a $(r, z)$ section of the basic state potential vorticity $2(B u / R o)(\bar{Q}-1)$ for (a) $m=2, b=0, B u=0.1$ and (b) $m=2, b=0, B u=0.3$.

$(b=0)$. For a Gaussian base state, the right hand side of condition (A.4) is

$$
\frac{R o}{2} \mathrm{e}^{-\left(r^{2}+z^{2}\right)}\left(z_{\text {top }}-z_{\text {bottom }}\right) \quad \text { at } \quad z=\left\{\begin{array}{l}
z_{\text {top }}, \\
z_{\text {bottom }}
\end{array}\right.
$$

whose maximum amplitude is $1.4 \times 10^{-15}$ for $R o=1$ and $z_{\text {top }}=-z_{\text {bottom }}=6$. Figure 3 shows that $|\operatorname{Re}\{c\}|>0.1$ for the most unstable azimuthal mode $m=2$ for all $B u$. Thus the ratio of the right and left hand sides of condition (A.4) is $1.4 \times 10^{-14}$, which ascertains that condition (A.4) holds for the Gaussian base state and domain height considered hereafter.

The critical levels in the $(r, z)$ plane, overlayed on top of the basic state potential vorticity field, are shown in figures 4(a) and (b) for the four most unstable eigenmodes of $m=2$, for respectively $B u=0.1$ and $B u=0.3$. The critical levels correspond to locations where the angular phase speed $c$ is equal to the base state angular velocity

$$
\frac{1}{r} \frac{\partial \bar{\psi}}{\partial r}=\operatorname{Re}\{c\}
$$

The critical levels are located well outside the vortex core, and also outside the area of maximal vertical and/or radial shear of the swirl velocity of the vortex. Note that the critical level of the first four leading eigenmodes are closely spaced, with a vertical spacing between levels in the range of non-dimensional 
$\delta z \approx 0.02-0.10$, which is much smaller than the base state vortex size (figures $4(\mathrm{a}, \mathrm{b})$ ). The spatial pattern of the hierarchy of unstable modes (not shown) for the continuous stratification does not follow a simple rule, such as being ordered according to the number of zeros of the real part of the eigenfunction (similarly to the Oscillation Theorem in the Sturm-Liouville theory, as in the two-layer case of Benilov 2003), but displays instead local modifications of their streamfunction curvature that are not simply related to their potential vorticity field. The spatial structure of the streamfunction of the leading unstable eigenmode in the $(r, z)$ plane for both co- and counter-rotating vortices is shown in figures $5(\mathrm{a}, \mathrm{b})$, with the position of their respective critical level overlaid. The potential vorticity extrema of the leading unstable eigenmode is shown on the right panel : their location exactly coincides with the position of critical level in the case of thin lenses $(B u<1)$. We have checked that, in that case, potential vorticity extrema correspond to extrema of the vortex stretching component of potential vorticity, $f^{2}\left(\psi_{z}^{\prime} / N^{2}\right)_{z}$, rather than the relative vorticity component $\nabla^{2} \psi^{\prime}$. Therefore the critical layer will correspond to the location of a strong density gradient, i.e. a density interface. However, in actual flows, such density interfaces are in general the spatial boundaries of homogenized regions : both phenomena of density interfaces and homogenized regions are usually found together in the perturbed state after the perturbation has evolved and this will give rise to persistent layering.

On the other hand, in the case of a tall lens (with $B u>1$ ), its destabilization (third row of figure $5(\mathrm{a})$ for $B u=2.0)$ involves extrema in potential vorticity that are dominated by relative vorticity, and are located nearer the core of the base state vortex.

\subsection{Initial-value simulations}

The stability of co-rotating vortices has also been investigated through a time integration of the linearized equation (3) initialized with random disturbances, using the spectral code of Hua and Haidvogel (1986) with a resolution of $256^{2} \times 480$ vertical levels. The integration shows the growth of transient features as well as the final dominance of the exponential modes which have been identified in the previous section. The disturbances have been projected onto azimuthal mode $m=2$ and results are shown in figure 6 at different stages of the growth of disturbances. Orr-like mechanisms cause the transient growth of regularly spaced extrema in potential vorticity with vertical scales that become thinner with time (Orr 1907, McWilliams et al. 2003). At the late stages of the integration, there is a clear evidence of the dominance of the fastest growing exponential mode at the periphery of the vortex for $\sqrt{r^{2}+z^{2}} \approx 1.6$, with weaker secondary extrema closely spaced in its immediate vicinity.

\section{Discussion and summary}

This work addresses the linear, exponentially-growing dynamics underlying the phenomenon of layering, involving the ubiquituous formation of sharp vertical interfaces of temperature anomalies, preferentially located at the periphery of energetic vortices, well outside the vortex core both in the radial and vertical directions. This was performed by numerically computing the slow modes of destabilization of a vortex lens in a continuously stratified rotating fluid. They were found to be associated with the critical layer instabilities such as discussed by Benilov (2003) for a two-layer configuration. Although the most unstable mode has a vertical size that is comparable to the vortex height, the relative locations of the critical levels pertaining to the successively fastest growing modes are quite closely spaced, at intervals along the axial direction that are much smaller than the vortex height.

Orders of magnitude for the vertical spacing between successive critical levels in the parameter range of oceanic intrathermocline eddies, with $L \approx 20-30 \mathrm{~km}, N / f \approx 50, B u \approx 0.1-1, H=\sqrt{B u}(N / f) L \approx$ $200-600 \mathrm{~m}$, corresponds to a typical vertical spacing in the range of $4-60 \mathrm{~m}$ (using the result of section 3.2 that non-dimensional spacing between critical levels $\delta z \approx 0.02-0.10$ ). This matches the range of observed vertical scales for the layering at the periphery of oceanic intrathermocline eddies (Quentel et al. 2010).

Our results suggest that the sharpness of the interfaces between layers can be linked to the existence of critical layers of the leading most unstable modes. The exponential instability does not yield a definite 

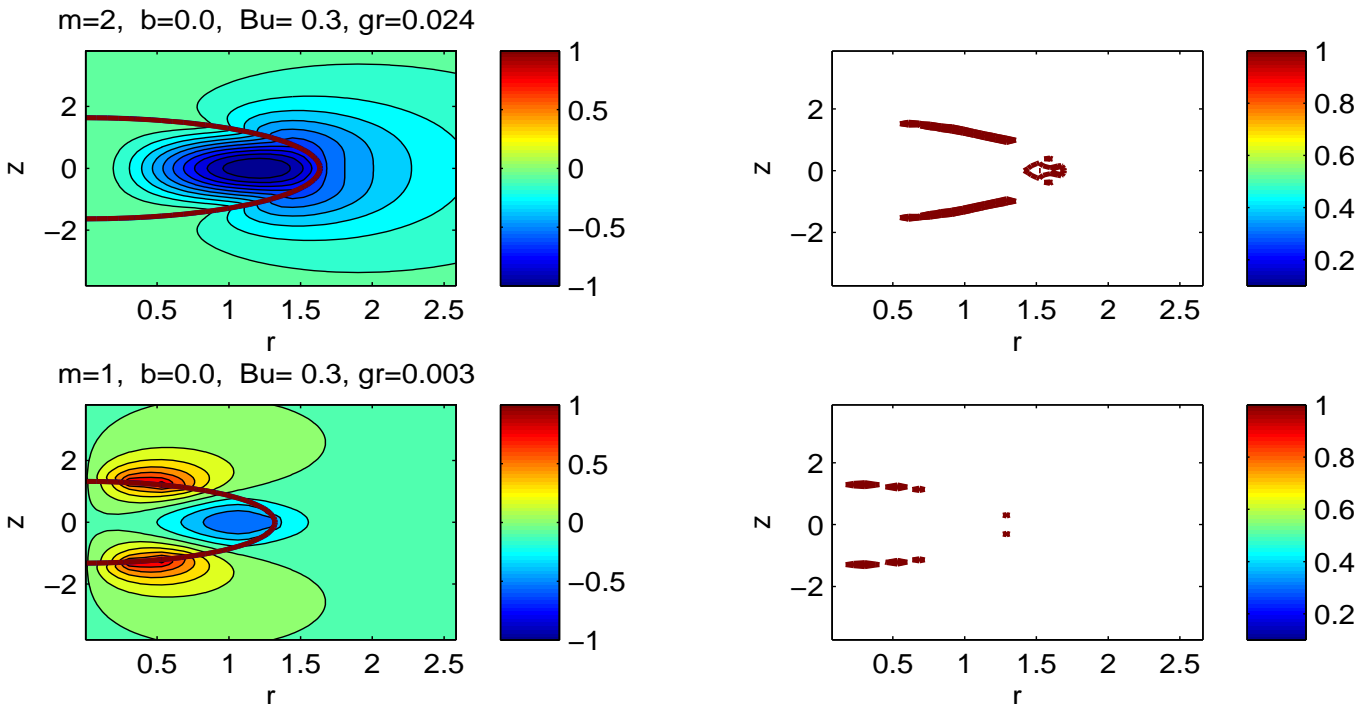

$m=1, b=0.0, B u=2.0, g r=0.021$
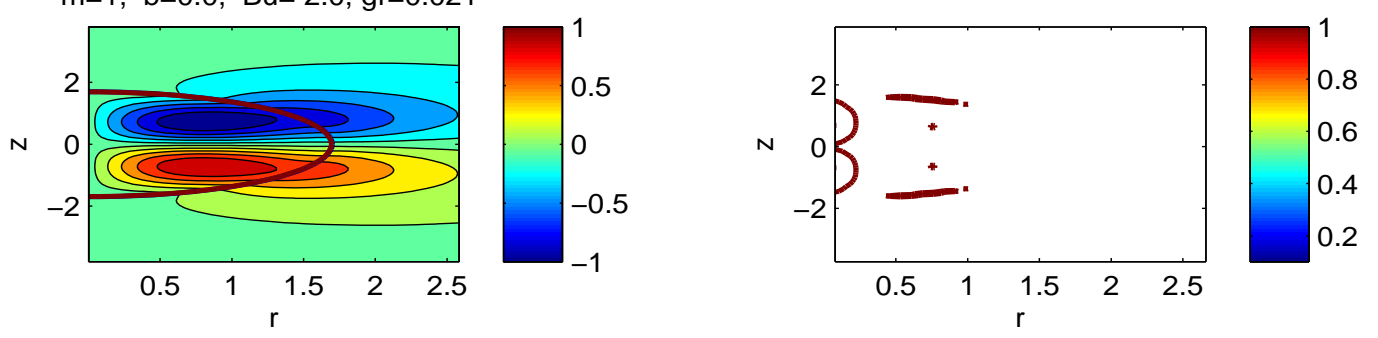

(a)
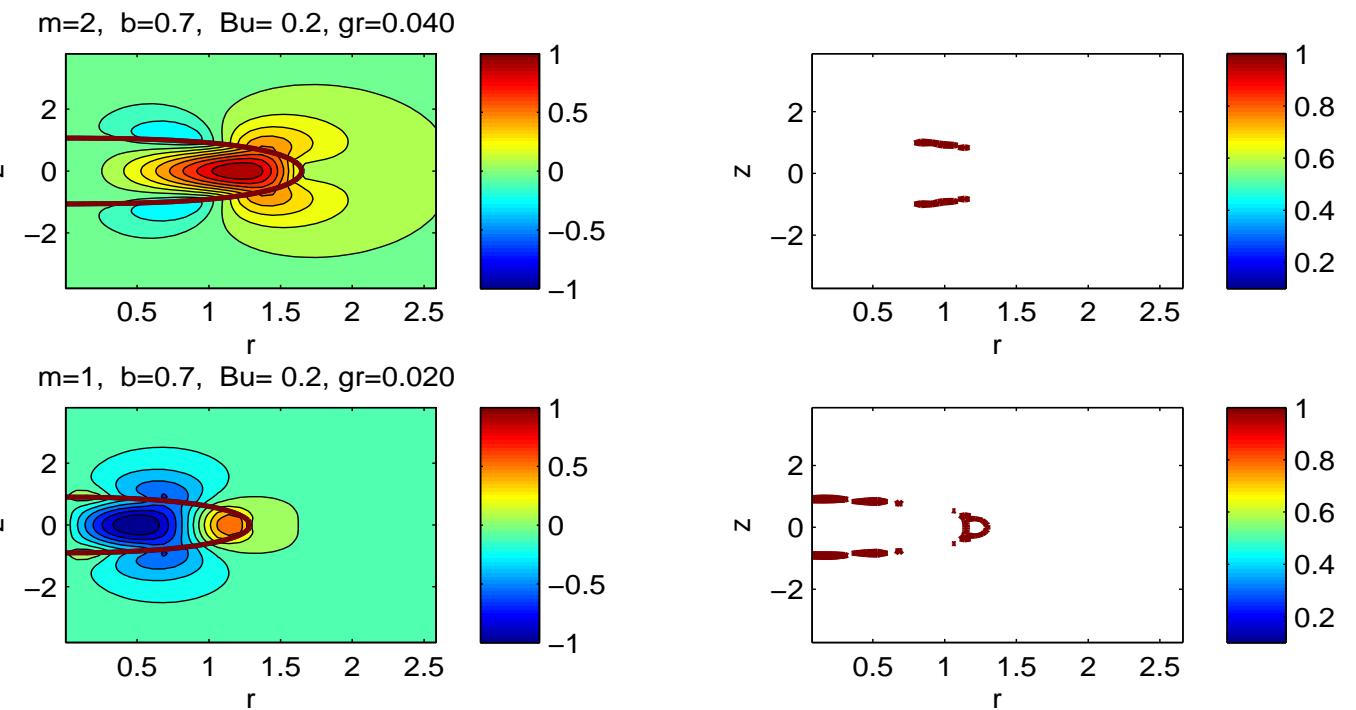

$m=1, b=0.7, B u=0.2, g r=0.029$
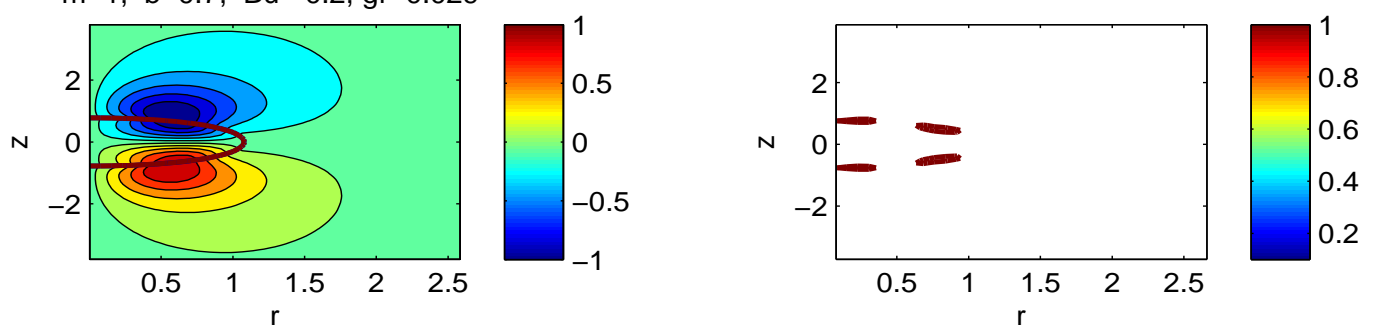

(b)

Figure 5. Spatial pattern of the leading unstable eigenmode for different azimutal modes : streamfunction field with critical level overlayed (left) and corresponding potential vorticity extrema (right). Eigenmodes correspond to both a co-rotating and counter-rotating basic state [respectively, (a) $b=0.0$, (b) $b=0.7$. The growth rate is indicated on top of each panel. 

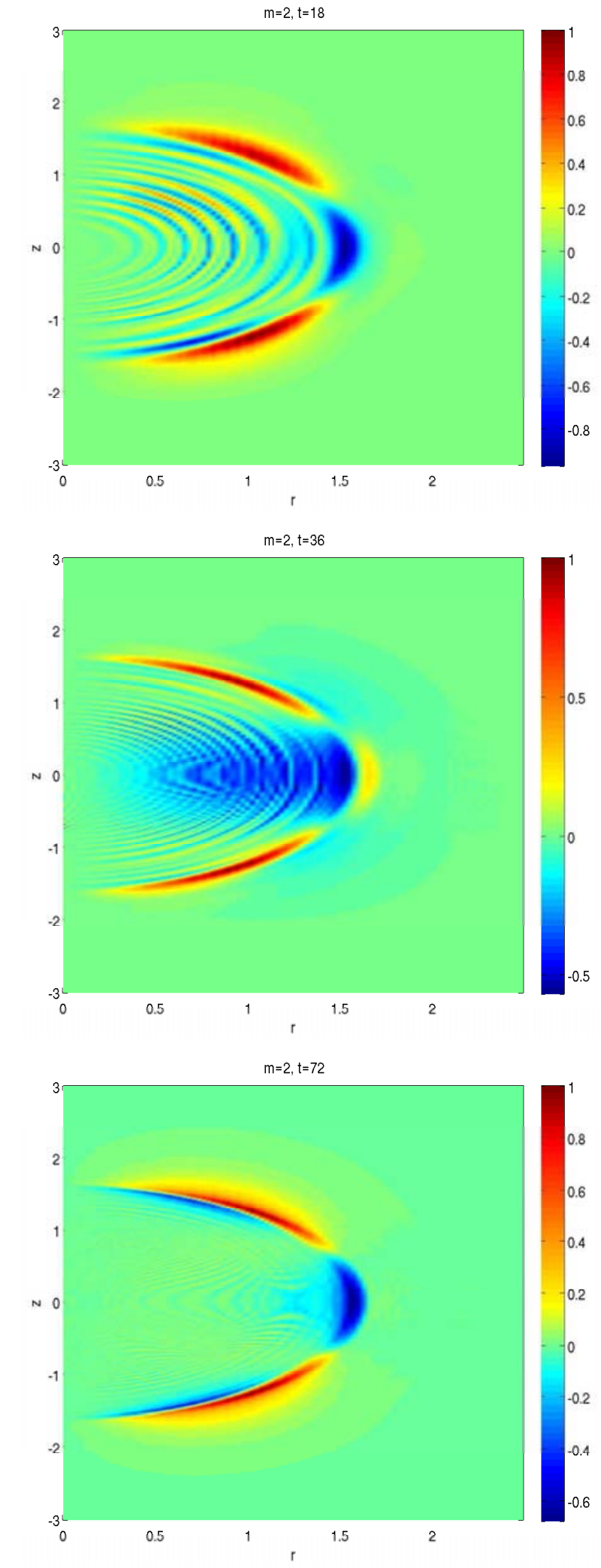

Figure 6. Forward time integration of linearized potential vorticity for a Gaussian lens with $b=0.0, B u=0.3$. Projection of potential vorticity disturbances on azimuthal mode $m=2$ at successive times (in rotation periods units). Each instantaneous plot has a color map normalized to the extremum value of the field.

$m=2, t=18$ 
scale selection, but the locations of the critical layers of the leading modes could favor the growth of sharp interfaces with vertical scales characteristic of layering.

Furthermore, figure 3 shows that the leading unstable mode for a given $B u$, namely azimuthal mode $m=2$ (resp. $m=1$ ) for $B u<1$ (resp. $B u>1$ ), always corresponds to a location of the critical layer outside the vortex core, with an amplitude of the real part of the angular velocity that is less than 0.2. For the case of a Gaussian lens, independently of the nature of the dominant instability mechanism (baroclinic instability for $B u<1$ and barotropic instability for $B u>1$ ), we thus find that the peripheral location of critical layers appears generic. Such results, obtained for a Gaussian lens in a continuously stratified flow, differ from the asymptotic results of Benilov (2003) who found, for a setup corresponding to a vortex in a two-layer model with a thin upper layer, that only baroclinic instability would favor unstable modes that are slow enough for their critical layer to be located outside the vortex core.

Finally, the appendix documents the location of the critical layer of a purely barotropic Gaussian vortex and a purely vertically-sheared Gaussian zonal flow. The latter case shows that only disturbances with long wavelengths will have critical layer located outside the vortex core and that moreover there are only 2 unstable modes in the case of pure baroclinic instability. This suggests that the layering which we attribute to the critical layers of the successively fastest growing modes found for the Gaussian lens (equation 7) exist because the radial confinement of the lens induces a larger number of unstable eigenmodes with similar values of their angular phase speeds.

\section{Références}

Armi, L., Hebert, D., Oakey, N., Price, J.F., Richardson, P.I., Rossby, H.T. and Ruddick, B., Two years in a life of a Mediterranean salt lens. J. Phys. Oceanogr. 1989, 19, 354-370.

Baey, J. and Carton, X., Vortex multipoles in two-layer rotating shallow-water flows. J. Fluid Mech. 2002, 460, 151-175.

Balmforth, N., Smith, S.G.L. and Young, W.R., Disturbing vortices. J. Fluid Mech. 2001, 426, 95.

Benilov, E.S., Instability of quasigeostrophic vortices in a two-layer ocean with a thin upper layer. J. Fluid Mech. 2003, 475, 303-331.

Benilov, E.S. and Flanagan, J., The effect of ageostrophy on the stability of vortices in a two-layer ocean.. Ocean Mod. 2008, 23, 49-58.

Biescas, B., Sallars, V., Pelegri, J.L., Machin, F., Carbonell, R., Buffett, G., Danobeitia, J.J. and Calahorrano, A., Imaging meddy finestructure using multichannel seismic reflection data. Geophys. Res. Lett. 2008, 35, L033971.

Dewar, W.K. and Killworth, P., On the stability of oceanic rings. J. Phys. Oceanogr. 1995, 25, 1467-1487.

Dewar, W.K., Killworth, P. and Blundell, J., Primitive-equation instability of wide oceanic rings. II. Numerical studies of ring stability. J. Phys. Oceanogr. 1999, 29, 1744-1758.

Drijfhout, S., Why anticyclones can split. J. Phys. Oceanogr. 2003, 33, 1579-1591.

Dritschel, D.G. and de la Torre Juarez, M., The instability and breakdown of tall columnar vortices in a quasi-geostrophic fluid. J. Fluid Mech. 1996, 328, 129-160.

Flierl, G.R., On the instability of geostrophic vortices. J. Fluid. Mech. 1988, 197, 349-388.

Gent, P. and McWilliams, J.C., The instability of barotropic circular vortices. Geophys. Astrophys. Fluid Dyn. 1986, 35, 209-233.

Helfrich, K.R. and Send, U., Finite-amplitude evolution of two-layer geostrophic vortices. J. Fluid. Mech. 1988, 197, 331-348.

Hua, B.L. and Haidvogel, D.B., Numerical simulations of the vertical structure of quasi-geostrophic turbulence. J. Atmos.Sci. 1986, 43, 2923-2936.

Katsman, C.A., der Vaart, P.C.V., Dijkstra, H.A. and de Ruijter, W.P.M., Stability of multilayer ocean vortices; a parameter study including realistic Gulf Stream and Agulhas Rings. J. Phys. Oceanogr. 2003, 33, 1197-1216.

McWilliams, J., Graves, L.P. and Montgomery, M.T., A Formal Theory for Vortex Rossby Waves and Vortex Evolution. Geophys. Astr. Fluid Dyn. 2003, 4, 275-309.

Menesguen, C., Hua, B., Papenberg, C., Klaeschen, D., Geli, L. and Hobbs, R., Effect of bandwidth on 
seismic imaging of rotating stratified turbulence surrounding an anticyclonic eddy from field data and numerical simulations. Geophys. Res. Let. 2009a, 36, doi :10.1029/2009GL039951.

Menesguen, C., Hua, B. and Schopp, R., Intermittent layering in the Atlantic equatorial deep jets. J. Mar. Res. 2009b, 67, 347-360.

Olson, D.B., Rings in the ocean. Ann. Rev. Earth Planet. Sci. 1991, 19, 283-311.

Orr, W.M.F., The stability or instability of the steady motions of a perfect liquid and of a viscous liquid. Part II : A viscous fluid. Proc. R. Irish Acad. 1907, A 27, 69-138.

Papenberg, C., Klaeschen, D., Krahmann, G. and Hobbs, R.W., Ocean temperature and salinity inverted from combined hydrographic and seismic data. Geophys. Res. Let. 2010, 37, doi :10.1029/2009GL042115.

Pedlosky, J., Ocean Circulation theory, 1996 (Springer-Verlag).

Quentel, E., Carton, X., Gutscher, M.A. and Hobbs, R., Detecting and characterizing mesoscale and submesoscale structures of Mediterranean water from joint seismic and hydrographic measurements in the Gulf of Cadiz. Geophys. Res. Let. 2010, 37, doi :10.1029/2010GL042766.

Reinaud, J.N. and Carton, X., The stability and the nonlinear evolution of quasi-geostrophic hetons. $J$. Fluid Mech. 2009, 636, 109-135.

Ruddick, B.R. and Richards, K.J., Oceanic thermohaline intrusions : observations. Prog. In Oceanogr. 2003, 56, 499-527.

Tychensky, A., Les Meddies : observation et modlisation de leurs interactions avec le courant des Aores., 1999 (Ph.D Univ. Toulouse III).

\section{Acknowledgements}

This work has been made possible through grant 96130 of IDRIS (France) and through the M.O.U. signed between IFREMER and JAMSTEC, enabling access to Earth Simulator (Yokohama, Japan). We would like to thank Sylvie Le Gentil for her help with the parrallel quasi-geostrophic code. H.Y. Nguyen acknowledges support from "Conseil General du Finisterre" during the completion of this work.

\section{Annexe A}

Rigid lid boundary conditions at the ocean surface and bottom actually correspond to

$$
\Psi_{z t}+J\left(\Psi, \Psi_{z}\right)=0
$$

and are exactly satisfied by the base state vortex by virtue of its axial symmetry. The boundary condition at $z=z_{\text {top }}$ and $z=z_{\text {bottom }}$ for the disturbance streamfunction is

$$
\left(c-\frac{\bar{\psi}_{r}}{r}\right) \psi_{z}+\frac{\psi}{r} \bar{\psi}_{z r}=0 .
$$

Equation (A.1) can be reduced to

$$
\psi_{z}=0,
$$

if

$$
\operatorname{Re}\{c\} \gg\left|\frac{\bar{\psi}_{r z}}{r} \frac{\psi}{\psi_{z}}\right|,\left|\frac{\bar{\psi}_{r}}{r}\right| .
$$

An upper bound of the first term on the right hand side of (A.3) corresponds to a vertical scale of the perturb ation as tall as the full domain height,

$$
\left|\frac{\bar{\psi}_{r z}}{r} \frac{\psi}{\psi_{z}}\right|<\left|\frac{\bar{\psi}_{r}}{r}\left(z_{\text {top }}-z_{\text {bottom }}\right)\right| \text {, }
$$


recalling that $z_{\text {top }}-z_{\text {bottom }}$ is the dimensionless domain height (scaled by the base state vortex thickness). Thus a sufficient condition is that

$$
|\operatorname{Re}\{c\}| \gg \max \left(\left|\frac{\bar{\psi}_{r}}{r}\right|\right)\left(z_{\text {top }}-z_{\text {bottom }}\right) \quad \text { at } \quad z=\left\{\begin{array}{l}
z_{\text {top }}, \\
z_{\text {bottom }} .
\end{array}\right.
$$

This relation obviously covers the two conditions of (A.3) if $\left(z_{\text {top }}-z_{\text {bottom }}\right)>1$. The condition (A.4) is verified provided the top and bottom boundaries are sufficiently distant from the eddy center for a base state vortex whose amplitude is rapidly decaying away from the origin. This was checked explicitly a posteriori in section 3 .

\section{Annexe B Pure barotropic instability of Gaussian column vortex in a stratifed medium}

The case of a barotropic column vortex with a Gaussian radial profile for the streamfunction field

$$
\bar{\psi}(r)=\mathrm{e}^{-r^{2}}
$$

has been studied by Gent and McWilliams (1986) and their figure 1(b) shows the real part of the propagation speed for the most unstable mode $\operatorname{Re}\{m c\}$, which magnitude is less than 0.25 for azimuthal mode $m=1$ (which has the largest growth rate), thus corresponding to a critical layer outside the vortex core.

\section{Annexe C Pure barotroclinic instability of a Gaussian vertically sheared flow}

The limit of pure baroclinic instability can be addressed by considering the base state

$$
\bar{U}(z)=-\mathrm{e}^{-z^{2}},
$$

whose meridional gradient of ambiant potential vorticy is

$$
\frac{\mathrm{d} \bar{Q}}{\mathrm{~d} y}=-\frac{\mathrm{d}^{2} \bar{U}}{\mathrm{~d} z^{2}}
$$

Looking for disturbances such as

$$
\psi^{\prime}=F(z) \mathrm{e}^{\mathrm{i} k(x-c t)} \cos l y
$$

leads to the eigenvalue problem

$$
(\bar{U}-c)\left(\frac{\mathrm{d}^{2} F}{\mathrm{~d} z^{2}}-K^{2} F\right)+\frac{\mathrm{d} \bar{Q}}{\mathrm{~d} y} F=0
$$

with $K^{2}=k^{2}+l^{2}$ and boundary conditions $\mathrm{d} F / \mathrm{d} z=0$ at the surface and bottom of the domain.

Only two unstable modes are found and figure $\mathrm{C} 1$ displays the growth rate and the real part of the propagation speed as a function of zonal wavenumber $k$, for the case of a prescribed value of $l=0.1$. Figure $\mathrm{C} 1$ (b) reveals that for the most unstable mode, the longest wavelengths will correspond to slowly propagating disturbances $(\approx 0.15)$ so that their critical layer will lie outside the vortex core. The variation of the propagation speed with horizontal wavenumber can be rationalized by multiplying equation (C.2) by the complex conjugate of $F$ and add to the result the product of $F$ with the complex conjugate of C.2. 


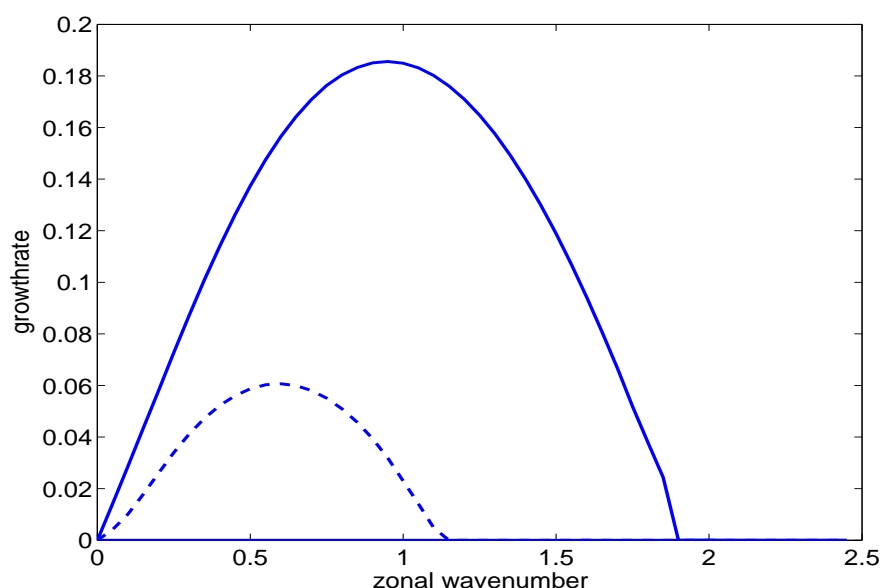

(a)

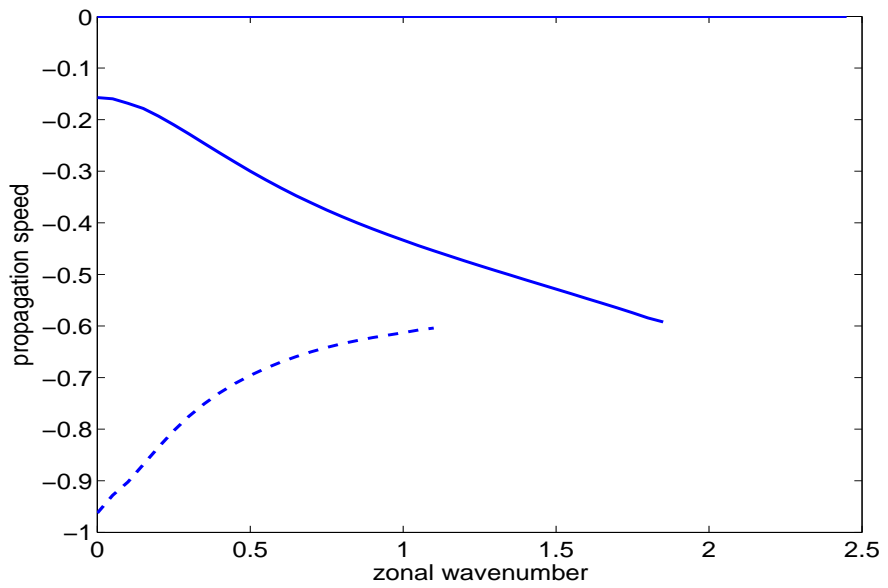

(b)

Figure C1. (a) Growth rate of the only two unstable modes for the pure baroclinic instability case of base state (C.1); (b) real part of their propagation speed as a function of zonal wavenumber

The expression is integrated with respect to $z$ on the domain height yielding

$$
\operatorname{Re}(c)=\frac{\int \bar{U}\left|\frac{\mathrm{d} F}{\mathrm{~d} z}\right|^{2} \mathrm{~d} z+\int \frac{\mathrm{d}^{2} \bar{U}}{\mathrm{~d} z^{2}}|F|^{2} \mathrm{~d} z+K^{2} \int \bar{U}|F|^{2} \mathrm{~d} z}{\int\left|\frac{\mathrm{d} F}{\mathrm{~d} z}\right|^{2} \mathrm{~d} z+K^{2} \int|F|^{2} \mathrm{~d} z} .
$$

